\title{
ON EXACT STRONG LAWS OF LARGE NUMBERS UNDER GENERAL DEPENDENCE CONDITIONS
}

\author{
BY
}

ANDRÉ ADLER (Chicago) ANd PRZEMYSŁAW MATUŁA (Lublin)

\begin{abstract}
We study the almost sure convergence of weighted sums of dependent random variables to a positive and finite constant, in the case when the random variables have either mean zero or no mean at all. These are not typical strong laws and they are called exact strong laws of large numbers. We do not assume any particular type of dependence and furthermore consider sequences which are not necessarily identically distributed. The obtained results may be applied to sequences of negatively associated random variables.
\end{abstract}

2010 AMS Mathematics Subject Classification: Primary: 60F15; Secondary: 60F05.

Key words and phrases: Strong law of large numbers, almost sure convergence, slowly varying functions, negative association.

\section{INTRODUCTION}

Let $\left\{X_{n}, n \geqslant 1\right\}$ be a sequence of independent random variables with the same distribution as the r.v. $X$. In the case $\mathbb{E}|X|<\infty$ and $\mathbb{E} X \neq 0$, from the classical Kolmogorov strong law of large numbers it follows that

$$
\lim _{n \rightarrow \infty} \frac{1}{n \mathbb{E} X} \sum_{k=1}^{n} X_{k}=1 \text { almost surely. }
$$

On the other hand, Chow and Robbins [4] proved that in the case $\mathbb{E}|X|=\infty$, there is no sequences $\left\{M_{n}, n \geqslant 1\right\}$ such that

$$
\lim _{n \rightarrow \infty} \frac{1}{M_{n}} \sum_{k=1}^{n} X_{k}=1 \text { almost surely. }
$$

A similar result was obtained by Maller [11] when $\mathbb{E} X=0$. Thus, we focus on the case $\mathbb{E} X=0$ or $\mathbb{E}|X|=\infty$, asking if there exist sequences of real numbers 
$\left\{a_{n}, n \geqslant 1\right\}$ and $\left\{b_{n}, n \geqslant 1\right\}$ such that

$$
\lim _{n \rightarrow \infty} \frac{1}{b_{n}} \sum_{k=1}^{n} a_{k} X_{k}=1 \text { almost surely. }
$$

Problems of this kind are called exact strong laws of large numbers and have been widely studied by Adler (see [1] where further references may be found). The assumption of independence in (ㅍ.J) and (ㅍ.2) was relaxed by Etemadi [5] and Hong and Park [ [ $]$ to pairwise independence. While there is a lot of results implying ([L.J) under dependence assumptions, the conditions for ([L.2) and ([L.3) remain unknown for dependent sequences and for sequences of r.v.s with different distributions. The goal of this paper is to fill this gap.

We do not make any particular assumptions on the dependence structure of the r.v.s. Instead of this we shall make use of the following conditions. The first one is related to the second Borel-Cantelli lemma.

ASSUMPTION 1.1. Let $\left\{Y_{n}, n \geqslant 1\right\}$ be a sequence of r.v.s with the same distribution as the r.v. $Y$ with the following property. For any sequence $\left\{b_{n}, n \geqslant 1\right\}$ of real numbers, if $\sum_{n=1}^{\infty} \mathbb{P}\left(|Y|>b_{n}\right)=\infty$, then

$$
\mathbb{P}\left(\lim \sup \left\{\left|Y_{n}\right|>b_{n}\right\}\right)=\mathbb{P}\left(\left|Y_{n}\right|>b_{n}, \text { i.o. }\right)=1 .
$$

The other assumption is related to the so-called second Kolmogorov-type maximal inequality for moments.

ASSUMPTION 1.2. Let $\left\{Y_{n}, n \geqslant 1\right\}$ be a centered sequence of square-integrable r.v.s such that

$$
\mathbb{P}\left(\max _{m \leqslant n \leqslant M}\left|S_{n}-S_{m}\right| \geqslant \varepsilon\right) \leqslant \frac{C}{\varepsilon^{2}} \sum_{k=m+1}^{M} \operatorname{Var}\left(Y_{k}\right)
$$

for some $C>0$, every $0 \leqslant m \leqslant M$, with $S_{n}=\sum_{k=1}^{n} Y_{k}, Y_{0}=0$ and $S_{0}=0$.

In order to discuss when the above conditions could be satisfied, let us recall some definitions. The notion of negatively quadrant dependent (NQD) r.v.s is due to Lehmann [10].

DEFINITION 1.1. We say that $\left\{Y_{n}, n \geqslant 1\right\}$ is a sequence of pairwise negatively quadrant dependent random variables if

$$
\mathbb{P}\left(Y_{k} \leqslant x, Y_{m} \leqslant y\right) \leqslant \mathbb{P}\left(Y_{k} \leqslant x\right) \mathbb{P}\left(Y_{m} \leqslant y\right)
$$

for all $k, m \in \mathbb{N}, k \neq m$ and $x, y \in \mathbb{R}$.

The more restrictive concept was introduced by Joag-Dev and Proschan [8]. 
DEFINITION 1.2. We say that $\left\{Y_{n}, n \geqslant 1\right\}$ is a sequence of negatively associated random variables if for any finite, nonempty and disjoint subsets $A, B \subset \mathbb{N}$ and coordinatewise nondecreasing functions $f: \mathbb{R}^{\text {Card } A} \rightarrow \mathbb{R}$ and $g: \mathbb{R}^{\text {Card } B} \rightarrow \mathbb{R}$, the inequality

$$
\operatorname{Cov}\left(f\left(Y_{i} ; i \in A\right), g\left(Y_{j} ; j \in B\right)\right) \leqslant 0
$$

holds whenever this covariance is finite.

A weaker definition of asymptotically almost negatively associated (AANA) random variables was introduced by Chandra and Ghosal in [3].

DEFINITION 1.3. A sequence $\left\{Y_{n}, n \geqslant 1\right\}$ of r.v.s is called asymptotically almost negatively associated if there exists a sequence $\{q(m) ; m \geqslant 1\}$ of nonnegative numbers, converging to zero, such that

$$
\begin{aligned}
& \operatorname{Cov}\left(f\left(Y_{m}\right), g\left(Y_{m+1}, \ldots, Y_{m+k}\right)\right) \\
& \quad \leqslant q(m)\left(\operatorname{Var}\left(f\left(Y_{m}\right)\right) \operatorname{Var}\left(g\left(Y_{m+1}, \ldots, Y_{m+k}\right)\right)\right)^{1 / 2}
\end{aligned}
$$

for all $m, k \geqslant 1$ and for all coordinatewise nondecreasing continuous functions $f$ and $g$, whenever the right-hand side of (IL.4) is finite.

Finally, let us recall the notion of $\rho^{-}$-mixing sequences which was introduced by Zhang and Wang (see [14]).

DEFINITION 1.4. A sequence $\left\{Y_{n}, n \geqslant 1\right\}$ of r.v.s is called $\rho^{-}$-mixing if

$$
\rho^{-}(s)=\sup \left\{\rho^{-}(S, T): S, T \subset \mathbb{N}, \operatorname{dist}(S, T) \geqslant s\right\} \rightarrow 0, \quad s \rightarrow \infty,
$$

where

$$
\rho^{-}(S, T):=\max \left(0 ; \sup \left\{\frac{\operatorname{Cov}\left(f\left(Y_{i}, i \in S\right), g\left(Y_{j}, j \in T\right)\right)}{\sqrt{\operatorname{Var}\left(f\left(Y_{i}, i \in S\right)\right) \operatorname{Var}\left(g\left(Y_{j}, j \in T\right)\right)}}\right\}\right),
$$

and the supremum (in the definition of the mixing coefficient $\rho^{-}(\cdot, \cdot)$ ) runs over all coordinatewise nondecreasing functions $f$ and $g$.

From the properties of the above-mentioned dependence concepts it follows that Assumption 1.2 is satisfied by negatively associated sequences, AANA with $\sum_{m=1}^{\infty} q^{2}(m)<\infty$ and $\rho^{-}$-mixing sequences with summable mixing coefficients (see [6]). In Section 2 we shall prove that Assumption 1.1 is satisfied by sequences of pairwise NQD r.v.s. It should be noted that negatively associated sequences are AANA and $\rho^{-}$-mixing as well as they are pairwise NQD. Among examples of negatively associated r.v.s we can find negatively correlated Gaussian sequences, furthermore the considered classes of dependent variables are closed under monotonic transformation of the r.v.s. This property will be used in construction examples in the last section of the paper. 
In this paper we study sequences of r.v.s with different distributions and for this reason we need the following assumption.

ASSUMPTION 1.3. We say that a sequence $\left\{X_{n}, n \geqslant 1\right\}$ of r.v.s has uniformly equivalent tails to the tails of the r.v. $X$ if

$$
\begin{aligned}
& \sup _{n \in \mathbb{N}}\left|\frac{\mathbb{P}\left(X_{n}<-x\right)}{\mathbb{P}(X<-x)}-1\right| \rightarrow 0, \quad x \rightarrow+\infty, \\
& \sup _{n \in \mathbb{N}}\left|\frac{\mathbb{P}\left(X_{n}>x\right)}{\mathbb{P}(X>x)}-1\right| \rightarrow 0, \quad x \rightarrow+\infty,
\end{aligned}
$$

where $\mathbb{P}(X>x)=0$ iff $\mathbb{P}\left(X_{n}>x\right)=0$, as well as $\mathbb{P}(X<-x)=0$ iff $\mathbb{P}\left(X_{n}<-x\right)=0$. In such cases we understand that " $0 / 0 "=1$.

The paper is organized as follows. In Section 2 we extend the results of Chow and Robbins [4] and Hong and Park [ 7 ] on exact sequences to r.v.s satisfying Assumption 1.1. In Sections 3 and 4 the results of Adler [I] are extended to sequences satisfying Assumptions 1.2 and 1.3. The examples are discussed in Section 5. The proofs are essentially similar to those known from the case of independent random variables, but for completeness we present most of the details.

\section{EXACT SEQUENCES}

In what follows we proceed as in the papers of Chow and Robbins [4] and Hong and Park [7], the proofs are essentially unchanged, and therefore we omit the details. Instead of making the assumption of the independence of the random variables under consideration, we shall assume that the sequence satisfies the second Borel-Cantelli lemma in the form described in Assumption 1.1.

LEMmA 2.1. If $\left\{b_{n} / n ; n \geqslant 1\right\}$ is positive and non-decreasing, and Assumption 1.1 is satisfied, then $\sum_{n=1}^{\infty} \mathbb{P}\left(|X|>b_{n}\right)=\infty$ implies

$$
\mathbb{P}\left(\limsup _{n \rightarrow \infty}\left|X_{n}\right| / b_{n}=\infty\right)=1 .
$$

Lemma 2.2. If $\left\{b_{n} / n ; n \geqslant 1\right\}$ is positive and non-decreasing, and Assumption 1.1 is satisfied, then $\sum_{n=1}^{\infty} \mathbb{P}\left(|X|>b_{n}\right)<\infty$ implies

$$
\mathbb{P}\left(\lim _{n \rightarrow \infty}\left|X_{n}\right| / b_{n}=0\right)=1 .
$$

In the next lemmas we deal with the partial sums $S_{n}=\sum_{k=1}^{n} X_{k}$ of the sequence $\left\{X_{n} ; n \geqslant 1\right\}$.

LEMmA 2.3. If $\left\{b_{n} / n ; n \geqslant 1\right\}$ is positive and non-decreasing, and Assumption 1.1 is satisfied, then $\sum_{n=1}^{\infty} \mathbb{P}\left(|X|>b_{n}\right)=\infty$ implies

$$
\mathbb{P}\left(\limsup _{n \rightarrow \infty}\left|S_{n}\right| / b_{n}=\infty\right)=1 \text {. }
$$


LEMMA 2.4. If $\left\{b_{n} / n ; n \geqslant 1\right\}$ is positive and non-decreasing, Assumption 1.1 is satisfied and $\mathbb{E}|X|=\infty$, then for any subsequence $\left\{n_{k} ; k \geqslant 1\right\}$ the condition $\sum_{n=1}^{\infty} \mathbb{P}\left(|X|>b_{n}\right)<\infty$ implies $\mathbb{P}\left(\liminf _{k \rightarrow \infty}\left|S_{n_{k}}\right| / b_{n_{k}}=0\right)=1$.

The main result of this section is an improvement of Theorem 1 in [ 7$]$. The proof relies on the aforementioned Lemmas 2.1-2.4 and follows the lines of [7], therefore the details will be omitted.

THEOREM 2.1. If Assumption 1.1 is satisfied and $\mathbb{E}|X|=\infty$, then for any sequence $\left\{b_{n} ; n \geqslant 1\right\}$ of constants,

$$
\text { either } \mathbb{P}\left(\liminf _{n \rightarrow \infty}\left|S_{n} / b_{n}\right|=0\right)=1 \text { or } \mathbb{P}\left(\limsup _{n \rightarrow \infty}\left|S_{n} / b_{n}\right|=\infty\right)=1 \text {, }
$$

and in consequence $\mathbb{P}\left(\lim _{n \rightarrow \infty} S_{n} / b_{n}=1\right)=0$.

From the above theorem we can derive a supplement to the strong law of large numbers for pairwise NQD random variables proved by Matuła in [12].

THEOREM 2.2. Let $\left\{X_{n} ; n \geqslant 1\right\}$ be a sequence of pairwise negatively quadrant dependent random variables with the same distribution as the r.v. $X$. Then the following two conditions are equivalent:

$$
\begin{gathered}
\mathbb{P}\left(\lim _{n \rightarrow \infty} S_{n} / n=c\right)=1 \quad \text { for some constant } c \in \mathbb{R} ; \\
\mathbb{E}|X|<\infty .
\end{gathered}
$$

If (2.2) holds, then $c=\mathbb{E} X$. If ([2.2) does not hold, i.e. $\mathbb{E}|X|=\infty$, then for any sequence $\left\{b_{n} ; n \geqslant 1\right\}$ of constants $\mathbb{P}\left(\lim _{n \rightarrow \infty} S_{n} / b_{n}=1\right)=0$.

Pro of. We have to prove that the sequence of pairwise NQD r.v.s satisfies Assumption 1.1. In the case that infinitely many of $b_{n}$ 's are negative, we have automatically $\sum_{n=1}^{\infty} \mathbb{P}\left(|X|>b_{n}\right)=\infty$ as well as $\mathbb{P}\left(\left|X_{n}\right|>b_{n}\right.$, i.o. $)=1$, and therefore the implication stated in Assumption 1.1 is satisfied irrespective of the dependence structure of the sequence $\left\{X_{n} ; n \geqslant 1\right\}$. Thus we may and do assume that $b_{n} \geqslant 0$ for all $n \in \mathbb{N}$. Let us introduce the events $A_{n}=\left\{X_{n}>b_{n}\right\}$ and $B_{n}=$ $\left\{X_{n}<-b_{n}\right\}$. From the NQD property, for $n \neq m$, we have

$$
\begin{aligned}
& \mathbb{P}\left(A_{n} \cap A_{m}\right) \leqslant \mathbb{P}\left(A_{n}\right) \mathbb{P}\left(A_{m}\right), \\
& \mathbb{P}\left(B_{n} \cap B_{m}\right) \leqslant \mathbb{P}\left(B_{n}\right) \mathbb{P}\left(B_{m}\right) .
\end{aligned}
$$

From $\sum_{n=1}^{\infty} \mathbb{P}\left(|X|>b_{n}\right)=\infty$ we get either $\sum_{n=1}^{\infty} \mathbb{P}\left(A_{n}\right)=\infty$ or $\sum_{n=1}^{\infty} \mathbb{P}\left(B_{n}\right)$ $=\infty$. Thus by Lemma 6.2 , p. 200, in [113] we have $\mathbb{P}\left(\lim \sup A_{n}\right)=1$ or $\mathbb{P}\left(\lim \sup B_{n}\right)=1$, and consequently $\mathbb{P}\left(\left|X_{n}\right|>b_{n}\right.$, i.o. $)=1$. 


\section{EXACT STRONG LAWS - MEAN ZERO CASE}

In what follows, $\log x=\ln x$ is the natural logarithm with base $e$. In this section, we shall assume that

$$
\mathbb{E}|X|<\infty \quad \text { and } \quad \mathbb{E} X=0 .
$$

Adapting the notation of Klass and Teicher [9], we define the function

$$
\bar{\mu}(x)=\int_{x}^{\infty} \mathbb{P}(|X|>t) d t .
$$

We have also to assume that the random variable $X$ is unbounded (for convenience, at least unbounded from above). In this case,

$$
\mathbb{P}(|X|>t)>0 \quad \text { for each } t \geqslant 0 .
$$

Since $\bar{\mu}(0)=\mathbb{E}|X|>0$ and $\mathbb{P}(|X|>t)$ is monotone decreasing, we have:

- $\bar{\mu}(x)$ is decreasing to zero,

- $\bar{\mu}(x)>0$ for each $x \geqslant 0$,

- $\lim _{t \rightarrow \infty} t \mathbb{P}(|X|>t)=0$.

LEMma 3.1 (Lemma 1 in [⿴囗十 $]$ ). Assume that there exist a constant $B>1$ and a slowly varying function $L(x)$ such that, for each $x \geqslant 0$,

$$
B^{-1} L(x) \leqslant x \mathbb{P}(|X|>x) \leqslant B L(x) .
$$

Then

(a) $\lim _{x \rightarrow \infty} x \mathbb{P}(|X|>x) / \bar{\mu}(x)=0$,

(b) $\bar{\mu}(x)$ and $1 / \bar{\mu}(x)$ are slowly varying functions,

(c) $\lim _{x \rightarrow \infty} \log \bar{\mu}(x) / \log x=0$.

Now, let us define the following function, which is a slightly modified formula of that in [1]:

$$
C(x)=\frac{x}{\bar{\mu}(x) \log (x+e)}, \quad x \geqslant 0 .
$$

Let us observe that

$$
\frac{d C}{d x}>\frac{\bar{\mu}(x)\left(\log (x+e)-\frac{x}{x+e}\right)}{(\bar{\mu}(x) \log (x+e))^{2}} \geqslant \frac{1}{\bar{\mu}(x) \log ^{2}(x+e)}>0 ;
$$

moreover,

$$
\log C(x)=\left(1-\frac{\log \bar{\mu}(x)}{\log x}-\frac{\log \log (x+e)}{\log x}\right) \log x \rightarrow \infty \quad \text { as } x \rightarrow \infty,
$$


thus $C(x) \rightarrow \infty$ as $x \rightarrow \infty$. This means that $C:[0, \infty) \rightarrow[0, \infty)$ is a continuous, increasing bijection. Let us denote by $c(x)=C^{-1}(x)$ the inverse function. Define a sequence $\left\{c_{n}, n \geqslant 1\right\}$ by $c_{n}=c(n)$. It is immediate that

$$
\lim _{n \rightarrow \infty} c_{n}=\infty .
$$

From the definition, we obtain the following equality:

$$
c_{n}=n \bar{\mu}\left(c_{n}\right) \log \left(c_{n}+e\right) .
$$

The main property of the sequence $\left\{c_{n}, n \geqslant 1\right\}$ is described in the following lemma.

LEMMA 3.2 (Lemma 2 in [⿴囗十]). Under the assumptions of Lemma [.] we have

$$
\lim _{n \rightarrow \infty} \frac{\log c_{n}}{\log n}=1 .
$$

Let us define the measure of symmetry of the r.v. $X$ as the following coefficient (we assume that this limit exists):

$$
\bar{c}=\lim _{x \rightarrow \infty} \frac{\mathbb{E} X^{-} \mathbb{I}\left[X^{-}>x\right]}{\mathbb{E} X^{+} \mathbb{I}\left[X^{+}>x\right]},
$$

where $X^{+}=X \mathbb{I}[X>0]$ and $X^{-}=-X \mathbb{I}[X<0]$. Then $X=X^{+}-X^{-}$and $|X|=X^{+}+X^{-}$.

REMARK 3.1. As noted in [1] , if $\lim _{x \rightarrow \infty} \frac{\mathbb{P}(X<-x)}{\mathbb{P}(X>x)}=0$, then $\bar{c}=0$.

The properties of the truncated moments of the r.v.s $X, X^{+}, X^{-}$and $|X|$ will be collected in the next lemma. The proof is essentially included in the proof of Theorem 1 in [1]].

LEMMA 3.3. Under the assumptions of Lemma B.] we have

$$
\begin{gathered}
\lim _{x \rightarrow \infty} \frac{\mathbb{E}|X| \mathbb{I}[|X|>x]}{\bar{\mu}(x)}=1 ; \\
\lim _{x \rightarrow \infty} \frac{\mathbb{E} X^{+} \mathbb{I}\left[X^{+}>x\right]+\mathbb{E} X^{-} \mathbb{I}\left[X^{-}>x\right]}{\bar{\mu}(x)}=1 ; \\
\lim _{x \rightarrow \infty} \frac{\mathbb{E} X^{+} \mathbb{I}\left[X^{+}>x\right]+\mathbb{E} X^{-} \mathbb{I}\left[X^{-}>x\right]}{\mathbb{E} X^{+} \mathbb{I}\left[X^{+}>x\right]}=1+\bar{c} ; \\
\lim _{x \rightarrow \infty} \frac{\mathbb{E} X^{-} \mathbb{I}\left[X^{-}>x\right]}{\bar{\mu}(x)}=\frac{\bar{c}}{1+\bar{c}} ;
\end{gathered}
$$


(e) if, moreover, $\mathbb{E} X=0$, then

$$
\lim _{x \rightarrow \infty} \frac{\mathbb{E} X \mathbb{I}[|X| \leqslant x]}{\bar{\mu}(x)}=\frac{\bar{c}-1}{\bar{c}+1} .
$$

Now let us investigate the properties of sequences with uniformly equivalent tails, i.e. satisfying our Assumption 1.3. For any nonnegative random variable $Y$ we have the formula

$$
\mathbb{E} Y=x \mathbb{P}(Y>x)+\int_{x}^{\infty} \mathbb{P}(Y>t) d t
$$

and in consequence, for $x>0$,

$$
\begin{aligned}
\mathbb{E} X^{+} \mathbb{I}\left[X^{+}>x\right] & =x \mathbb{P}\left(X^{+}>x\right)+\int_{x}^{\infty} \mathbb{P}\left(X^{+}>t\right) d t \\
& =x \mathbb{P}(X>x)+\int_{x}^{\infty} \mathbb{P}(X>t) d t
\end{aligned}
$$

and

$$
\begin{aligned}
\mathbb{E} X^{-} \mathbb{I}\left[X^{-}>x\right] & =x \mathbb{P}\left(X^{-}>x\right)+\int_{x}^{\infty} \mathbb{P}\left(X^{-}>t\right) d t \\
& =x \mathbb{P}(-X>x)+\int_{x}^{\infty} \mathbb{P}(-X>t) d t .
\end{aligned}
$$

Further, under the assumption $\mathbb{E} X=0$ we have

$$
\mathbb{E} X \mathbb{I}[|X| \leqslant x]=-\mathbb{E} X \mathbb{I}[|X|>x]=-\mathbb{E} X^{+} \mathbb{I}\left[X^{+}>x\right]+\mathbb{E} X^{-} \mathbb{I}\left[X^{-}>x\right] .
$$

We shall examine the following quantity for large $x$ :

$$
\frac{\mathbb{E} X_{n} \mathbb{I}\left[\left|X_{n}\right| \leqslant x\right]}{\mathbb{E} X^{+} \mathbb{I}\left[X^{+}>x\right]}=\frac{-\mathbb{E} X_{n}^{+} \mathbb{I}\left[X_{n}^{+}>x\right]+\mathbb{E} X_{n}^{-} \mathbb{I}\left[X_{n}^{-}>x\right]}{\mathbb{E} X^{+} \mathbb{I}\left[X^{+}>x\right]} .
$$

From our assumptions, for any $\varepsilon>0$, there exists $x(\varepsilon)$ such that for any $x \geqslant x(\varepsilon)$ we have

$$
\begin{gathered}
\left|\frac{\mathbb{P}\left(X_{n}<-x\right)}{\mathbb{P}(X<-x)}-1\right|<\varepsilon \quad \text { for each } n \in \mathbb{N}, \\
\left|\frac{\mathbb{P}\left(X_{n}>x\right)}{\mathbb{P}(X>x)}-1\right|<\varepsilon \quad \text { for each } n \in \mathbb{N}, \\
\bar{c}-\varepsilon<\frac{\mathbb{E} X^{-} \mathbb{I}\left[X^{-}>x\right]}{\mathbb{E} X^{+} \mathbb{I}\left[X^{+}>x\right]}<\bar{c}+\varepsilon .
\end{gathered}
$$


Therefore,

$$
\begin{gathered}
(1-\varepsilon) \mathbb{P}(X>x)<\mathbb{P}\left(X_{n}>x\right)<(1+\varepsilon) \mathbb{P}(X>x), \\
(1-\varepsilon) \mathbb{P}(X<-x)<\mathbb{P}\left(X_{n}<-x\right)<(1+\varepsilon) \mathbb{P}(X<-x) .
\end{gathered}
$$

Thus,

$$
\frac{\mathbb{E} X_{n}^{+} \mathbb{I}\left[X_{n}^{+}>x\right]}{\mathbb{E} X^{+} \mathbb{I}\left[X^{+}>x\right]} \geqslant \frac{x(1-\varepsilon) \mathbb{P}(X>x)+(1-\varepsilon) \int_{x}^{\infty} \mathbb{P}(X>t) d t}{\mathbb{E} X^{+} \mathbb{I}\left[X^{+}>x\right]}=1-\varepsilon
$$

and

$$
\begin{aligned}
\frac{\mathbb{E} X_{n}^{-} \mathbb{I}\left[X_{n}^{-}>x\right]}{\mathbb{E} X^{+} \mathbb{I}\left[X^{+}>x\right]} & =\frac{x \mathbb{P}\left(X_{n}<-x\right)+\int_{x}^{\infty} \mathbb{P}\left(X_{n}<-t\right) d t}{\mathbb{E} X^{+} \mathbb{I}\left[X^{+}>x\right]} \\
& \leqslant \frac{(1+\varepsilon) \mathbb{E} X^{-} \mathbb{I}\left[X^{-}>x\right]}{\mathbb{E} X^{+} \mathbb{I}\left[X^{+}>x\right]} \leqslant(1+\varepsilon)(\bar{c}+\varepsilon) .
\end{aligned}
$$

Consequently, for $x \geqslant x(\varepsilon)$ and all $n \in \mathbb{N}$, we get

$$
\frac{\mathbb{E} X_{n} \mathbb{I}\left[\left|X_{n}\right| \leqslant x\right]}{\mathbb{E} X^{+} \mathbb{I}\left[X^{+}>x\right]} \leqslant-1+\varepsilon+(1+\varepsilon)(\bar{c}+\varepsilon) .
$$

Thus, for any $c_{n} \rightarrow \infty$,

$$
\limsup _{n \rightarrow \infty} \frac{\mathbb{E} X_{n} \mathbb{I}\left[\left|X_{n}\right| \leqslant c_{n}\right]}{\mathbb{E} X^{+} \mathbb{I}\left[X^{+}>c_{n}\right]} \leqslant \bar{c}-1 .
$$

Similarly we get

$$
\liminf _{n \rightarrow \infty} \frac{\mathbb{E} X_{n} \mathbb{I}\left[\left|X_{n}\right| \leqslant c_{n}\right]}{\mathbb{E} X^{+} \mathbb{I}\left[X^{+}>c_{n}\right]} \geqslant \bar{c}-1 .
$$

We have proved the following lemma.

LEMMA 3.4. Let $\left\{X_{n} ; n \geqslant 1\right\}$ be a sequence of r.v.s with uniformly equivalent tails to $X$, and $\left\{c_{n} ; n \geqslant 1\right\}$ be a sequence of real numbers such that $\lim _{n \rightarrow \infty} c_{n}=\infty$. Then

$$
\lim _{n \rightarrow \infty} \frac{\mathbb{P}\left(X_{n}>c_{n}\right)}{\mathbb{P}\left(X>c_{n}\right)}=\lim _{n \rightarrow \infty} \frac{\mathbb{P}\left(X_{n}<-c_{n}\right)}{\mathbb{P}\left(X<-c_{n}\right)}=\lim _{n \rightarrow \infty} \frac{\mathbb{P}\left(\left|X_{n}\right|>c_{n}\right)}{\mathbb{P}\left(|X|>c_{n}\right)}=1 ;
$$

in consequence, $\sum_{n=1}^{\infty} \mathbb{P}\left(\left|X_{n}\right|>c_{n}\right)<\infty$ iff $\sum_{n=1}^{\infty} \mathbb{P}\left(|X|>c_{n}\right)<\infty$. If, moreover, $\mathbb{E} X=0$ and (B.4) is satisfied, then

$$
\begin{gathered}
\lim _{n \rightarrow \infty} \frac{\mathbb{E} X_{n} \mathbb{I}\left[\left|X_{n}\right| \leqslant c_{n}\right]}{\mathbb{E} X^{+} \mathbb{I}\left[X^{+}>c_{n}\right]}=\bar{c}-1, \\
\lim _{n \rightarrow \infty} \frac{\mathbb{E} X_{n} \mathbb{I}\left[\left|X_{n}\right| \leqslant c_{n}\right]}{\mathbb{E} X \mathbb{I}\left[|X| \leqslant c_{n}\right]}=1,
\end{gathered}
$$


and, in consequence,

$$
\lim _{n \rightarrow \infty} \frac{\mathbb{E} X_{n} \mathbb{I}\left[\left|X_{n}\right| \leqslant c_{n}\right]}{\bar{\mu}\left(c_{n}\right)}=\frac{\bar{c}-1}{\bar{c}+1}
$$

An elementary lemma concerning convergence of real sequences will be needed in the proofs.

LeMmA 3.5. Let $\left\{A_{n} ; n \geqslant 1\right\},\left\{B_{n} ; n \geqslant 1\right\},\left\{\alpha_{n} ; n \geqslant 1\right\}$ and $\left\{\beta_{n} ; n \geqslant 1\right\}$ be sequences of positive real numbers such that $\lim _{n \rightarrow \infty} A_{n} / B_{n}=1$ and $\lim _{n \rightarrow \infty} \beta_{n}=\infty$. Then

$$
\lim _{n \rightarrow \infty} \frac{1}{\beta_{n}} \sum_{k=1}^{n} \alpha_{k} A_{k}=a
$$

for some constant a, iff

$$
\lim _{n \rightarrow \infty} \frac{1}{\beta_{n}} \sum_{k=1}^{n} \alpha_{k} B_{k}=a .
$$

THEOREM 3.1. If $\left\{Y_{n}, n \geqslant 1\right\}$ is a sequence of centered r.v.s which satisfy Assumption 1.2 and $\sum_{n=1}^{\infty} \operatorname{Var}\left(Y_{n}\right)<\infty$, then the series $\sum_{n=1}^{\infty} Y_{n}$ is almost surely convergent.

Pr o o f. We proceed as in the book of Petrov (see [113], pp. 204-205).

Let us define the normalizing sequences as $b_{n}=(\log n)^{b}$ for some $b>0$, and $a_{n}=b_{n} / c_{n}$ with $c_{n}$ defined previously.

THEOREM 3.2. Let $\left\{X_{n}, n \geqslant 1\right\}$ be a sequence of random variables satisfying Assumption 1.3 with $X$ such that (3.2) holds. Assume that for any nondecreasing bounded functions $\left\{f_{n}, n \geqslant 1\right\}$, the sequence $Y_{n}=f_{n}\left(X_{n}\right)-\mathbb{E} f_{n}\left(X_{n}\right)$ satisfies Assumption 1.2. If (B. I) and

$$
\sum_{n=1}^{\infty} \mathbb{P}\left(|X|>c_{n}\right)<\infty
$$

are satisfied, then

$$
\lim _{n \rightarrow \infty} \frac{1}{b_{n}} \sum_{k=1}^{n} a_{k} X_{k}=\frac{\bar{c}-1}{b(\bar{c}+1)} \text { almost surely. }
$$

Pro o f. Let us define the following functions $f_{k}(x)$ as the monotonic truncation:

$$
f_{k}(x)= \begin{cases}-c_{k}, & x<-c_{k} \\ x, & |x| \leqslant c_{k} \\ c_{k}, & x>c_{k}\end{cases}
$$


Then we have

$$
\begin{aligned}
& \frac{1}{b_{n}} \sum_{k=1}^{n} a_{k} X_{k}=\frac{1}{b_{n}} \sum_{k=1}^{n} a_{k}\left(f_{k}\left(X_{k}\right)-\mathbb{E} f_{k}\left(X_{k}\right)\right) \\
+ & \frac{1}{b_{n}} \sum_{k=1}^{n} a_{k} X_{k} \mathbb{I}\left(\left|X_{k}\right|>c_{k}\right)+\frac{1}{b_{n}} \sum_{k=1}^{n} a_{k} c_{k} \mathbb{I}\left(X_{k}<-c_{k}\right)-\frac{1}{b_{n}} \sum_{k=1}^{n} a_{k} c_{k} \mathbb{I}\left(X_{k}>c_{k}\right) \\
+ & \frac{1}{b_{n}} \sum_{k=1}^{n} a_{k} c_{k} \mathbb{P}\left(X_{k}>c_{k}\right)-\frac{1}{b_{n}} \sum_{k=1}^{n} a_{k} c_{k} \mathbb{P}\left(X_{k}<-c_{k}\right) \\
+ & \frac{1}{b_{n}} \sum_{k=1}^{n} a_{k} \mathbb{E} X_{k} \mathbb{I}\left(\left|X_{k}\right| \leqslant c_{k}\right) \\
:= & A_{1}+A_{2}+A_{3}+A_{4} .
\end{aligned}
$$

To prove that $A_{1} \rightarrow 0$ almost surely, as $n \rightarrow \infty$, we will show that $\sum_{n=1}^{\infty} \operatorname{Var}\left(f_{n}\left(X_{n}\right) / c_{n}\right)<\infty$ and apply Theorem 3.1 together with Kronecker's lemma. We have

$$
\sum_{n=1}^{\infty} \operatorname{Var}\left(\frac{f_{n}\left(X_{n}\right)}{c_{n}}\right) \leqslant \sum_{n=1}^{\infty} \frac{c_{n}^{2} \mathbb{P}\left(\left|X_{n}\right|>c_{n}\right)}{c_{n}^{2}}+\sum_{n=1}^{\infty} \frac{\mathbb{E} X_{n}^{2} \mathbb{I}\left(\left|X_{n}\right| \leqslant c_{n}\right)}{c_{n}^{2}} .
$$

From Assumption 1.3 and Lemma 3.4 it follows that there exists a constant $M>0$ such that $\mathbb{P}\left(\left|X_{n}\right|>c_{n}\right) \leqslant M \mathbb{P}\left(|X|>c_{n}\right)$ for all $n \geqslant 1$, and $\mathbb{P}\left(\left|X_{n}\right|>t\right) \leqslant$ $M \mathbb{P}(|X|>t)$ for all $t \geqslant 0$. Thus, by (B.5),

$$
\sum_{n=1}^{\infty} \mathbb{P}\left(\left|X_{n}\right|>c_{n}\right) \leqslant M \sum_{n=1}^{\infty} \mathbb{P}\left(|X|>c_{n}\right)<\infty .
$$

Moreover,

$$
\begin{aligned}
\sum_{n=1}^{\infty} \frac{\mathbb{E} X_{n}^{2} \mathbb{I}\left(|X| \leqslant c_{n}\right)}{c_{n}^{2}}=2 \sum_{n=1}^{\infty} \frac{1}{c_{n}^{2}} \int_{0}^{c_{n}} t \mathbb{P}\left(\left|X_{n}\right|>t\right) d t & \\
& \leqslant 2 B M \sum_{n=1}^{\infty} \frac{1}{c_{n}^{2}} \int_{0}^{c_{n}} L(t) d t
\end{aligned}
$$

since, by the Karamata theorem, $\int_{0}^{x} L(t) d t /(x L(x)) \rightarrow 1$ as $x \rightarrow \infty$, thus $\int_{0}^{c_{n}} L(t) d t /\left(c_{n} L\left(c_{n}\right)\right) \leqslant B_{1}$ for some $B_{1}$, it follows that the right-hand side of the inequality in (B.7) is less than or equal to

$$
2 B M B_{1} \sum_{n=1}^{\infty} \frac{L\left(c_{n}\right)}{c_{n}} \leqslant 2 B^{2} M B_{1} \sum_{n=1}^{\infty} \mathbb{P}\left(|X|>c_{n}\right)<\infty .
$$

The almost sure convergence $A_{2} \rightarrow 0$ as $n \rightarrow \infty$ follows from the first BorelCantelli lemma, which is a consequence of (B.5) and Lemma B.4. 
From the relation (B.6) it follows that $\sum_{n=1}^{\infty} \mathbb{P}\left(X_{n}>c_{n}\right)<\infty$ as well as $\sum_{n=1}^{\infty} \mathbb{P}\left(X_{n}<-c_{n}\right)<\infty$, so the Kronecker lemma implies $A_{3} \rightarrow 0$ as $n \rightarrow \infty$.

It remains to investigate $A_{4}=\frac{1}{b_{n}} \sum_{k=1}^{n} a_{k} \mathbb{E} X_{k} \mathbb{I}\left(\left|X_{k}\right| \leqslant c_{k}\right)$ or, by Lemmas [3.3-3.5, equivalently, $\frac{1}{b_{n}} \sum_{k=1}^{n} a_{k} \bar{\mu}\left(c_{k}\right)$, but it goes along the lines of the paper by Adler [1].

\section{EXACT STRONG LAWS - INFINITE MEAN CASE}

In this section we assume that $\mathbb{E}|X|=\infty$. Define the function

$$
\mu(x)=\int_{0}^{x} \mathbb{P}(|X|>t) d t .
$$

Then $X$ is unbounded, and in this case $\mathbb{P}(|X|>t)>0$ for each $t \geqslant 0$ (for convenience, we assume that it is at least unbounded from above) and $\mu(x) \rightarrow \infty$ as $x \rightarrow \infty$.

It may be useful later on to observe that for a nonnegative r.v. $Y \geqslant 0$ and $x>0$ we have $\mathbb{E} Y \mathbb{I}[Y \leqslant x]=-x \mathbb{P}(Y>x)+\int_{0}^{x} \mathbb{P}(Y>t) d t$. Thus,

$$
\begin{gathered}
\mathbb{E}|X| \mathbb{I}[|X| \leqslant x]=-x \mathbb{P}(|X|>x)+\int_{0}^{x} \mathbb{P}(|X|>t) d t, \\
\mu(x)=\int_{0}^{x} \mathbb{P}(|X|>t) d t=\mathbb{E}|X| \mathbb{I}[|X| \leqslant x]+x \mathbb{P}(|X|>x) .
\end{gathered}
$$

LEMMA 4.1 (Lemma 4 in [⿴囗十]). Assume that there exist a constant $B>1$ and a slowly varying function $L(x)$ such that (B.2) holds. Then

(a) $\lim _{x \rightarrow \infty} x \mathbb{P}(|X|>x) / \mu(x)=0$;

(b) $\mu(x)$ and $1 / \mu(x)$ are slowly varying functions;

(c) $\lim _{x \rightarrow \infty} \log \mu(x) / \log x=0$.

From the above lemma it follows that there exists $x(L)>0$ depending on the function $L$ and the distribution of $X$ such that, for $x \geqslant x(L)$,

$$
\frac{x \mathbb{P}(|X|>x)}{\mu(x)} \leqslant \frac{1}{2} .
$$

Now, let us define the function

$$
C(x)=\frac{x}{\mu(x) \log (x+e)} \quad \text { for } x \geqslant x(L)
$$

and linear between $C(0)=0$ and $C(x(L))>0$, i.e. for $0 \leqslant x \leqslant x(L)$. Let us observe that

$$
\frac{d C}{d x}>\frac{\frac{1}{2} \log 2}{\mu(x) \log ^{2}(x+e)}>0
$$


since the denominator attains its minimum value at $e$. Moreover, for $x \geqslant x(L)$,

$$
\log C(x)=\left(1-\frac{\log \mu(x)}{\log x}-\frac{\log \log (x+e)}{\log x}\right) \log x \rightarrow \infty \quad \text { as } x \rightarrow \infty,
$$

thus $C(x) \rightarrow \infty$ as $x \rightarrow \infty$. This means that $C:[0, \infty) \rightarrow[0, \infty)$ is a continuous, increasing bijection. Let us denote by $c(x)=C^{-1}(x)$ the inverse function. Define a sequence $\left\{c_{n}, n \geqslant 1\right\}$ by $c_{n}=c(n)$. It is immediate that $\lim _{n \rightarrow \infty} c_{n}=\infty$. From the definition, the following equality holds for sufficiently large $n$ :

$$
c_{n}=n \mu\left(c_{n}\right) \log \left(c_{n}+e\right) .
$$

The main property of the sequence $\left\{c_{n}, n \geqslant 1\right\}$ is described in the following lemma.

LEMMA 4.2 (Lemma 5 in [⿴囗⿴囗十)]). Under the assumptions of Lemma 4.1$]$ we have

$$
\lim _{n \rightarrow \infty} \frac{\log c_{n}}{\log n}=1 \text {. }
$$

Let us define the measure of symmetry of the r.v. $X$ as the following coefficient (we assume that this limit exists):

$$
c=\lim _{x \rightarrow \infty} \frac{\mathbb{E} X^{-} \mathbb{I}\left[X^{-} \leqslant x\right]}{\mathbb{E} X^{+} \mathbb{I}\left[X^{+} \leqslant x\right]},
$$

where $X^{+}=X \mathbb{I}[X>0]$ and $X^{-}=-X \mathbb{I}[X>0]$. Then $X=X^{+}-X^{-}$and $|X|=X^{+}+X^{-}$.

LEMMA 4.3 (Lemma 6 in [i]]). Under the assumptions of Lemma 4.1, if $\lim _{x \rightarrow \infty} \mathbb{P}(X<-x) / \mathbb{P}(X>x)=0$, then $c=0$.

Again the properties of the truncated moments of the r.v.s $X, X^{+}, X^{-}$and $|X|$ will be collected in the next lemma. The proof is essentially included in the proof of Theorem 2 in [四].

LEMMA 4.4. Under the assumptions of Lemma A.] we have

(d) $\lim _{x \rightarrow \infty} \frac{\mathbb{E} X^{-} \mathbb{I}\left[X^{-} \leqslant x\right]}{\mu(x)}=\frac{c}{1+c} \quad$ and $\quad \lim _{x \rightarrow \infty} \frac{\mathbb{E} X^{+} \mathbb{I}\left[X^{+} \leqslant x\right]}{\mu(x)}=\frac{1}{1+c}$;

$$
\lim _{x \rightarrow \infty} \frac{\mathbb{E} X \mathbb{I}[|X| \leqslant x]}{\mu(x)}=\frac{1-c}{1+c} .
$$


LEMMA 4.5. Let $\left\{X_{n} ; n \geqslant 1\right\}$ be a sequence of r.v.s with uniformly equivalent tails to $X$ with $\mathbb{E} X=\infty$ and satisfying (4.2). If $\left\{c_{n} ; n \geqslant 1\right\}$ is a sequence of real numbers such that $\lim _{n \rightarrow \infty} c_{n}=\infty$, then

$$
\lim _{n \rightarrow \infty} \frac{\mathbb{E} X_{n} \mathbb{I}\left[\left|X_{n}\right| \leqslant c_{n}\right]}{\mu\left(c_{n}\right)}=\frac{1-c}{1+c} .
$$

P r o of. In view of Lemma $4.4(\mathrm{e})$ it suffices to show that

$$
\lim _{n \rightarrow \infty} \frac{\mathbb{E} X_{n} \mathbb{I}\left[\left|X_{n}\right| \leqslant c_{n}\right]-\mathbb{E} X \mathbb{I}\left[|X| \leqslant c_{n}\right]}{\mu\left(c_{n}\right)}=0 .
$$

For any $\varepsilon>0$, we choose $x(\varepsilon)$ as in the considerations prior to Lemma B.4. Then, for $x \geqslant x(\varepsilon)$ and all $n \in \mathbb{N}$, we get the upper bound

$$
\begin{aligned}
& \mathbb{E} X_{n} \mathbb{I}\left[\left|X_{n}\right| \leqslant x\right]-\mathbb{E} X \mathbb{I}[|X| \leqslant x] \\
& =\mathbb{E} X_{n}^{+} \mathbb{I}\left[X_{n}^{+} \leqslant x\right]-\mathbb{E} X_{n}^{-} \mathbb{I}\left[X_{n}^{-} \leqslant x\right]-\mathbb{E} X^{+} \mathbb{I}\left[X^{+} \leqslant x\right]+\mathbb{E} X^{-} \mathbb{I}\left[X^{-} \leqslant x\right] \\
& =-x \mathbb{P}\left(X_{n}^{+}>x\right)+\int_{0}^{x} \mathbb{P}\left(X_{n}^{+}>t\right) d t+x \mathbb{P}\left(X_{n}^{-}>x\right)-\int_{0}^{x} \mathbb{P}\left(X_{n}^{-}>t\right) d t \\
& +x \mathbb{P}\left(X^{+}>x\right)-\int_{0}^{x} \mathbb{P}\left(X^{+}>t\right) d t-x \mathbb{P}\left(X^{-}>x\right)+\int_{0}^{x} \mathbb{P}\left(X^{-}>t\right) d t \\
& \leqslant-(1-\varepsilon) x \mathbb{P}\left(X^{+}>x\right)+x \mathbb{P}\left(X^{+}>x\right)+(1+\varepsilon) x \mathbb{P}\left(X^{-}>x\right)-x \mathbb{P}\left(X^{-}>x\right) \\
& +\int_{0}^{x(\varepsilon)} \mathbb{P}\left(X_{n}^{+}>t\right) d t+\int_{x(\varepsilon)}^{x} \mathbb{P}\left(X_{n}^{+}>t\right) d t-\int_{0}^{x} \mathbb{P}\left(X^{+}>t\right) d t \\
& -\int_{0}^{x(\varepsilon)} \mathbb{P}\left(X_{n}^{-}>t\right) d t-\int_{x(\varepsilon)}^{x} \mathbb{P}\left(X_{n}^{-}>t\right) d t+\int_{0}^{x} \mathbb{P}\left(X^{-}>t\right) d t \\
& \leqslant \varepsilon x \mathbb{P}(|X|>x)+x(\varepsilon)+(1+\varepsilon) \int_{0}^{x} \mathbb{P}\left(X^{+}>t\right) d t-\int_{0}^{x} \mathbb{P}\left(X^{+}>t\right) d t \\
& -(1-\varepsilon) \int_{x(\varepsilon)}^{x} \mathbb{P}\left(X^{-}>t\right) d t+\int_{0}^{x} \mathbb{P}\left(X^{-}>t\right) d t \\
& =\varepsilon x \mathbb{P}(|X|>x)+x(\varepsilon)+\varepsilon \int_{0}^{x} \mathbb{P}\left(X^{+}>t\right) d t \\
& -(1-\varepsilon) \int_{0}^{x} \mathbb{P}\left(X^{-}>t\right) d t+(1-\varepsilon) \int_{0}^{x(\varepsilon)} \mathbb{P}\left(X^{-}>t\right) d t+\int_{0}^{x} \mathbb{P}\left(X^{-}>t\right) d t \\
& \leqslant \varepsilon x \mathbb{P}(|X|>x)+(2-\varepsilon) x(\varepsilon)+\varepsilon \int_{0}^{x} \mathbb{P}(|X|>t) d t .
\end{aligned}
$$


Since $\mu\left(c_{n}\right) \rightarrow \infty$, by Lemma 4.1$]$ we get

$$
\limsup _{n \rightarrow \infty} \frac{\mathbb{E} X_{n} \mathbb{I}\left[\left|X_{n}\right| \leqslant c_{n}\right]-\mathbb{E} X \mathbb{I}\left[|X| \leqslant c_{n}\right]}{\mu\left(c_{n}\right)} \leqslant \varepsilon .
$$

Similarly we obtain the lower bound and the bound for lim inf.

As in the previous section we define $b_{n}=\log ^{b} n$ for some $b>0$, and $a_{n}=$ $b_{n} / c_{n}$. With such weights we get the following theorem.

THEOREM 4.1. Let $\left\{X_{n}, n \geqslant 1\right\}$ be a sequence of random variables satisfying Assumption 1.3 with $X$ such that (B.2) holds. Assume that, for any nondecreasing bounded functions $\left\{f_{n}, n \geqslant 1\right\}$, the sequence $Y_{n}=f_{n}\left(X_{n}\right)-\mathbb{E} f_{n}\left(X_{n}\right)$ satisfies Assumption 1.2. If $\mathbb{E}|X|=\infty$ and (3.5) holds, then

$$
\lim _{n \rightarrow \infty} \frac{1}{b_{n}} \sum_{k=1}^{n} a_{k} X_{k}=\frac{1-c}{b(c+1)} \text { almost surely. }
$$

Pro of. The proof is similar to that of the previous theorem. We split the sum in the same way, and deduce that $A_{1}, A_{2}, A_{3} \rightarrow 0$ in the same way. To study the convergence of $A_{4}$ we apply Lemma 4.5 , where it is proved that

$$
\frac{\mathbb{E} X_{n} \mathbb{I}\left[\left|X_{n}\right| \leqslant c_{n}\right]}{\mu\left(c_{n}\right)} \rightarrow \frac{1-c}{1+c} \quad \text { as } n \rightarrow \infty,
$$

and we proceed as in [1]].

\section{EXAMPLES}

A starting point in constructing our examples will be a sequence of negatively associated r.v.s. We extend the example presented in [6] and construct an example of a sequence of negatively associated r.v.s with the same distribution or different distributions but satisfying Assumption 1.3, for which the exact strong law may be applied.

EXAMPLE 5.1. Let us consider a Gaussian sequence $\left\{\xi_{n} ; n \geqslant 1\right\}$ such that $\xi_{n}$ 's have the standard normal distribution and $\operatorname{Cov}\left(\xi_{i}, \xi_{j}\right)=-a_{i j}, i \neq j, a_{i j} \geqslant 0$, with $\sum_{i=1}^{\infty} \sum_{j=1}^{\infty} a_{i j}=A<1$. These r.v.s are negatively correlated Gaussian and, according to Joag-Dev and Proschan [8], are negatively associated. To show that such a sequence exists it suffices to prove that the matrix $\mathbf{A}_{n}=\left[-a_{i j}\right]_{1 \leqslant i, j \leqslant n}$ with $a_{i i}=1$ is positive definite and it is indeed the covariance matrix of the vector $\left[\xi_{1}, \xi_{2}, \ldots, \xi_{n}\right]$. Let us write $\mathbf{A}_{n}=\mathbf{I}-\mathbf{B}$, where $\mathbf{I}$ is a unit matrix and $\mathbf{B}=\left[a_{i j}\right]$ with $a_{i i}=0$.

Let us focus on the matrix $\mathbf{B}$. We put $\mathbf{x}=\left[x_{1}, \ldots, x_{n}\right]$ and by the inequality $a_{i j} x_{i} x_{j} \leqslant a_{i j} \frac{x_{i}^{2}+x_{j}^{2}}{2}$, which holds for any $i, j$, we get, for any $1 \leqslant k \leqslant n$,

$$
\sum_{i=1}^{n} a_{i k} x_{i} x_{k} \leqslant \frac{1}{2} \sum_{i=1}^{n} a_{i k} x_{i}^{2}+\frac{1}{2} x_{k}^{2} \sum_{i=1}^{n} a_{i k} \leqslant \frac{A_{k}}{2}\|\mathbf{x}\|^{2}+\frac{1}{2} x_{k}^{2} A_{k} \leqslant A_{k}\|\mathbf{x}\|^{2},
$$


where $A_{k}=\sum_{i=1}^{\infty} a_{i k}$. Therefore,

$$
\mathbf{x} \mathbf{B} \mathbf{x}^{T}=\sum_{k=1}^{n} \sum_{i=1}^{n} a_{i k} x_{i} x_{k} \leqslant A\|\mathbf{x}\|^{2}
$$

and finally

$$
\mathbf{x} \mathbf{A}_{n} \mathbf{x}^{T}=\|\mathbf{x}\|^{2}-\mathbf{x} \mathbf{B} \mathbf{x}^{T} \geqslant(1-A)\|\mathbf{x}\|^{2}>0
$$

provided that $\mathbf{x} \neq \mathbf{0}$.

Now, let us denote by $\Phi$ the standard normal distribution, and let $F_{n}$ be any distribution with the quasi-inverse $F_{n}^{(-1)}$. Define $X_{n}=F_{n}^{(-1)}\left(\Phi\left(\xi_{n}\right)\right)$. Then $\left\{X_{n} ; k \geqslant 1\right\}$ is a sequence of negatively associated r.v.s with distributions $F_{n}$.

Let us note that all the examples of distributions given in [1] may be used in our Example 5.] to show how Theorems $\$ .2$ and 4.$]$ work in the case of identically distributed random variables.

Let us begin with a modification of one of those distributions.

EXAMPLE 5.2. To illustrate Theorem 4.], in Example [.], we take the distributions $F_{n}(x)=F(x)=1-\frac{1}{x+1}, x \geqslant 0$. Then

$$
\mu(x)=\int_{0}^{x} \mathbb{P}(|X|>t) d t=\int_{0}^{x} \frac{d t}{t+1}=\log (x+1),
$$

and for large $n$ we have $c_{n}=n \log \left(c_{n}+1\right) \log \left(c_{n}+e\right)$. Thus $c_{n} /\left(n \log ^{2} n\right) \rightarrow 1$, and by taking $b_{n}=\log ^{2} n$, we get $n a_{n}=n b_{n} / c_{n}=n \log ^{2} n / c_{n} \rightarrow 1$. Consequently,

$$
\frac{1}{\log ^{2} n} \sum_{k=1}^{n} \frac{X_{k}}{k} \rightarrow \frac{1}{2} \text { almost surely } \quad \text { as } n \rightarrow \infty .
$$

To explain how the symmetry coefficient $c$ works, let us extend the above example in the following way.

EXAMPLE 5.3. Let us consider the distribution function

$$
F(x)= \begin{cases}1-A /(1+x), & x \geqslant 0 \\ (1-A) /(1-x), & x<0\end{cases}
$$

with $A \in(0,1)$. Then

$$
\begin{aligned}
\mu(x) & =\int_{0}^{x} \mathbb{P}(|X|>t) d t=\int_{0}^{x} \mathbb{P}(X>t) d t+\int_{0}^{x} \mathbb{P}(X<-t) d t \\
& =\int_{0}^{x} \frac{A}{1+t} d t+\int_{0}^{x} \frac{1-A}{1+t} d t=\log (x+1) .
\end{aligned}
$$


Also, for $x>0$, we have

$$
\begin{aligned}
\mathbb{E} X^{+} \mathbb{I}\left[X^{+} \leqslant x\right] & =-x \mathbb{P}\left(X^{+}>x\right)+\int_{0}^{x} \mathbb{P}\left(X^{+}>t\right) d t \\
& =-x \mathbb{P}(X>x)+\int_{0}^{x} \mathbb{P}(X>t) d t \\
& =-\frac{A x}{1+x}+A \log (x+1),
\end{aligned}
$$

and similarly

$$
\mathbb{E} X^{-} \mathbb{I}\left[X^{-} \leqslant x\right]=-\frac{(1-A) x}{1+x}+(1-A) \log (x+1) .
$$

Therefore,

$$
c=\lim _{x \rightarrow \infty} \frac{\mathbb{E} X^{-} \mathbb{I}\left[X^{-} \leqslant x\right]}{\mathbb{E} X^{+} \mathbb{I}\left[X^{+} \leqslant x\right]}=\frac{1-A}{A} .
$$

Thus, as in Example 5.2, $c_{n} /\left(n \log ^{2} n\right) \rightarrow 1$, and by taking $b_{n}=\log ^{2} n$, we get $n a_{n}=n b_{n} / c_{n}=n \log ^{2} n / c_{n} \rightarrow 1$. Consequently, for a sequence $\left\{X_{n}, n \geqslant 1\right\}$ of negatively associated r.v.s with the same distribution function $F(x)$ defined in Example 5.11, we get

$$
\frac{1}{\log ^{2} n} \sum_{k=1}^{n} \frac{X_{k}}{k} \rightarrow A-\frac{1}{2} \text { almost surely } \quad \text { as } n \rightarrow \infty,
$$

since

$$
\frac{1-c}{1+c}=\frac{1-(1 / A-1)}{1+1 / A-1}=2 A-1 .
$$

In the last example we shall construct a sequence of non-identically distributed r.v.s satisfying Assumptions 1.2 and 1.3 for which Theorem 3.2 works.

EXAMPLE 5.4. Consider r.v.s $X$ and $X_{n}$ with the following distribution functions

$$
\begin{gathered}
F(x)= \begin{cases}0, & x<-2, \\
1 / 2, & -2 \leqslant x<0, \\
1-1 /\left((x+e) \log ^{2}(x+e)\right), & x \geqslant 0,\end{cases} \\
F_{n}(x)= \begin{cases}0, & x<-2, \\
1 / 2-1 /(10 n), & -2 \leqslant x<-1, \\
1 / 2+1 /(10 n), & -1 \leqslant x<0, \\
1-1 /\left((x+e) \log ^{2}(x+e)\right), & x \geqslant 0 .\end{cases}
\end{gathered}
$$


Then $\mathbb{E} X=\int_{0}^{\infty}(1-F(x)) d x-\int_{-\infty}^{0} F(x) d x=0$ as well as $\mathbb{E} X_{n}=0$. It is easy to see that Assumption 1.3 is satisfied. Furthermore, for $x \geqslant 2$ we have

$$
\mu(x)=\int_{x}^{\infty} \mathbb{P}(|X|>t) d t=\int_{x}^{\infty} \frac{d t}{(t+e) \log ^{2}(t+e)}=\frac{1}{\log (x+e)}
$$

and

$$
x \mathbb{P}(|X|>x)=\frac{x}{(x+e) \log ^{2}(x+e)} .
$$

On account of $C(x)=x /(\mu(x) \log (x+e))$, for $x \geqslant 2$ we get $C(x)=x$, and therefore we may put $c_{n}=n$. Moreover, $b_{n}=(\log n)^{b}$ with $b>0$ and $a_{n}=$ $(\log n)^{b} / n$. We have to check that $\sum_{n=1}^{\infty} \mathbb{P}\left(|X|>c_{n}\right)<\infty$. But for sufficiently large $n$ we have

$$
\mathbb{P}\left(|X|>c_{n}\right)=\mathbb{P}(X>n)=\frac{1}{(n+e) \log ^{2}(n+e)} .
$$

It is easy to see that for the distribution $F(x)$ we have

$$
\bar{c}=\lim _{x \rightarrow \infty} \frac{\mathbb{E} X^{-} \mathbb{I}\left[X^{-}>x\right]}{\mathbb{E} X^{+} \mathbb{I}\left[X^{+}>x\right]}=0,
$$

and therefore the sequence $\left\{X_{n} ; n \geqslant 1\right\}$ described in Example [5.] with such $F_{n}(x)$ and $F(x)$ satisfies the assumptions of Theorem 3.2, and the following weighted limit law holds:

$$
\lim _{n \rightarrow \infty} \frac{1}{\log ^{b} n} \sum_{k=1}^{n} \frac{(\log k)^{b}}{k} X_{k}=-\frac{1}{b} \text { almost surely. }
$$

\section{REFERENCES}

[1] A. Adler, Exact strong laws, Bull. Inst. Math. Acad. Sin. 28 (2000), pp. 141-166.

[2] N. H. Bingham, C. M. Goldie, and J. L. Teugels, Regular Variation, Cambridge University Press, Cambridge 1987.

[3] T. K. Chandra and S. Ghosal, The strong law of large numbers for weighted averages under dependence assumptions, J. Theoret. Probab. 9 (1996), pp. 797-809.

[4] Y. S. Chow and H. Robbins, On sums of independent random variables with infinite moments and "fair" games, Proc. Natl. Acad. Sci. USA 47 (1967), pp. 330-335.

[5] N. Etemadi, An elementary proof of the strong law of large numbers, Z. Wahrsch. Verw. Gebiete 55 (1981), pp. 119-122.

[6] I. Fazekas, P. Matuła, and M. Ziemba, A note on the weighted strong law of large numbers under general conditions, Publ. Math. Debrecen 9 (2017), pp. 373-386.

[7] D. H. Hong and J. M. Park, Exact sequences for sums of pairwise i.i.d. random variables, Bull. Korean Math. Soc. 30 (1993), pp. 167-170.

[8] K. Joag-Dev and F. Proschan, Negative association of random variables, with applications, Ann. Statist. 11 (1983), pp. 286-295. 
[9] M. Klass and H. Teicher, Iterated logarithm laws for asymmetric random variables barely with or without finite mean, Ann. Probab. 5 (1977), pp. 861-874.

[10] E. L. Lehmann, Some concepts of dependence, Ann. Math. Statist. 37 (1966), pp. 11371153.

[11] R. A. Maller, Relative stability and the strong law of large numbers, Z. Wahrsch. Verw. Gebiete 43 (1978), pp. 141-148.

[12] P. Matuła, A note on the almost sure convergence of sums of negatively dependent random variables, Statist. Probab. Lett. 15 (1992), pp. 209-213.

[13] V. V. Petrov, Limit Theorems of Probability Theory: Sequences of Independent Random Variables, Clarendon Press, Oxford 1995.

[14] L. X. Zhang and X. Y. Wang, Convergence rates in the strong laws of asymptotically negatively associated random fields, Appl. Math. J. Chinese Univ. 14 (1999), pp. 406-416.

André Adler

Department of Mathematics

Illinois Institute of Technology

Chicago, Illinois, 60616, USA

E-mail: adler@iit.edu
Przemysław Matuła Institute of Mathematics Marie Curie-Skłodowska University pl. Marii Curie-Skłodowskiej 1 20-031 Lublin, Poland

E-mail:matula@hektor.umcs.lublin.pl

Received on 4.8.2016;

revised version on 3.11.2016 